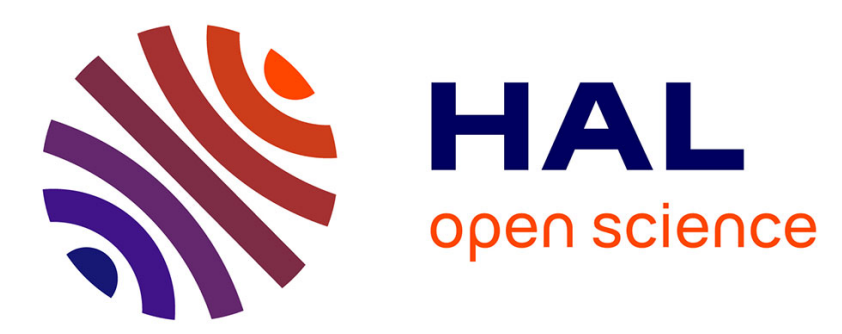

\title{
Hamiltonian structure of a drift-kinetic model and Hamiltonian closures for its two-moment fluid reductions
}

\author{
Emanuele Tassi
}

\section{To cite this version:}

Emanuele Tassi. Hamiltonian structure of a drift-kinetic model and Hamiltonian closures for its twomoment fluid reductions. The European Physical Journal D : Atomic, molecular, optical and plasma physics, 2014, 68, pp.196. hal-01053855

\section{HAL Id: hal-01053855 https://hal.science/hal-01053855}

Submitted on 2 Aug 2014

HAL is a multi-disciplinary open access archive for the deposit and dissemination of scientific research documents, whether they are published or not. The documents may come from teaching and research institutions in France or abroad, or from public or private research centers.
L'archive ouverte pluridisciplinaire HAL, est destinée au dépôt et à la diffusion de documents scientifiques de niveau recherche, publiés ou non, émanant des établissements d'enseignement et de recherche français ou étrangers, des laboratoires publics ou privés. 


\title{
Hamiltonian structure of a drift-kinetic model and Hamiltonian closures for its two-moment fluid reductions
}

\author{
E. $\operatorname{Tassi}^{1}$ \\ 1 Aix-Marseille Université, Université de Toulon, \\ CNRS, Centre de Physique Théorique, \\ UMR 7332, 13288 Marseille, France
}

\begin{abstract}
We address the problem of the existence of the Hamiltonian structure for an electrostatic driftkinetic model and for the related fluid models describing the evolution of the first two moments of the distribution function with respect to the parallel velocity. The drift-kinetic model, which accounts for background density and temperature gradients as well as polarization effects, is shown to possess a noncanonical Hamiltonian structure. The corresponding Poisson bracket is expressed in terms of the fluid moments and it is found that the set of functionals of the zero order moment forms a sub-algebra, thus automatically leading to a class of one-moment Hamiltonian fluid models. In particular, in the limit of weak spatial variations of the background quantities, the CharneyHasegawa-Mima equation, with its Hamiltonian structure, is recovered. For the set of functionals of the first two moments, which, unlike the case of the Vlasov equation, turns out not to form a sub-algebra, we look for closures that lead to a closed Poisson bracket restricted to this set of functionals. The constraint of the Jacobi identity turns out to select the adiabatic equation of state for an ideal gas with one-degree-of-freedom molecules, as the only admissible closure in this sense. When the so called $\delta f$ ordering is applied to the model, on the other hand, a Poisson bracket is obtained if the second order moment is a linear combination of the first two moments of the total distribution function. By means of this procedure, three-dimensional Hamiltonian fluid models that couple a generalized Charney-Hasegawa-Mima equation with an evolution equation for the parallel velocity are derived. Among these, a model adopted by Meiss and Horton ( Phys. Fluids 26, (1983) 990 ) to describe drift waves coupled to ion-acoustic waves, is obtained and its Hamiltonian structure is provided explicitly.
\end{abstract}




\section{INTRODUCTION}

Drift-kinetic models (see, e.g. Refs. [1-4]) represent a common and effective tool for investigating phenomena such as turbulence and transport in strongly magnetized plasmas, where the relevant dynamics takes place at frequencies much smaller than the ion cyclotron frequency based on the dominant component of the magnetic field. In many drift-kinetic models, the non-dissipative part of the so called drift-kinetic equation, takes the form of a Vlasov equation for a distribution function on a reduced phase space, devoid of the dependence on the gyration angle and labelled by the value of the particle magnetic moment, which is an adiabatic invariant for the guiding center dynamics. Assuming external electromagnetic fields, the characteristics of the drift-kinetic equation correspond then to the equations of motion for the particle guiding centers, and, unless forcing and/or dissipative effects are voluntarily included, these are supposed to form a Hamiltonian system. Such Hamiltonian character of the single guiding center dynamics, is supposed to be preserved also at the level of the field theory consisting of the drift-kinetic equation coupled self-consistently with electromagnetic fields through Maxwell's equations, which form a Hamiltonian system on their

own. In this respect, then, how such coupling is introduced is crucial in order to guarantee that the resulting field theory still possesses a Hamiltonian structure.

A further common reduction adopted in plasma physics, is that of considering fluid models obtained by taking moments of the drift-kinetic equation and imposing a closure relation. This leads to a system of evolution equations for the moments, whose reduced phase space includes only the spatial coordinates. The problem of identifying appropriate closures for fluid models has a long history in plasma physics and concerns important applications in both fusion and astrophysical plasmas (see, e.g. Refs. [5-9]). Of course, also the operation of taking moments and imposing a closure, does not in general preserve the original Hamiltonian character of the system and has to be done with care, if one wants to avoid the introduction of spurious dissipative effects.

For the Vlasov equation, the corresponding Poisson bracket [10, 11]was shown to trans- 
form into a Poisson bracket [12] (and thus preserve the Hamiltonian character of the system) when expressing it in terms of the moments [13] (see also Refs. [14-16] for further applications of this result).

For Hamiltonian electrostatic drift-kinetic systems the problem was recently addressed in Ref. [17], where the Poisson bracket of the drift-kinetic equation was derived in terms of the moments with respect to the parallel velocity, and the Hamiltonian structure of fluid models obtained from the first two moments was discussed. In particular, it was shown that, unlike the Vlasov case, the set of functionals of the zero and one order moment $P_{0}$ and $P_{1}$ is not closed under the Poisson bracket of two such functionals, because the latter turns out to depend also on the second order moment $P_{2}$. In order to identify closed Hamiltonian fluid models restricted to $P_{0}$ and $P_{1}$ we then looked for what we defined as Hamiltonian closures. A Hamiltonian closure is a function $\mathcal{F}$ such that inserting $P_{2}=\mathcal{F}\left(P_{0}, P_{1}\right)$ into the expression of the bracket between two functionals of $P_{0}$ and $P_{1}$, yields a Poisson bracket and therefore can lead to a Hamiltonian fluid model for $P_{0}$ and $P_{1}$. Assuming that $\mathcal{F}$ cannot depend on derivatives of $P_{0}$ and $P_{1}$ and on the spatial coordinates explicitly, it was shown that only the cold plasma closure $P_{2}=P_{1}^{2} / P_{0}$ is a Hamiltonian closure. If the $\delta f$ ordering is applied to the drift-kinetic equation, on the other hand, the Hamiltonian closure for the second order moment is given by a linear combination of the first two moments of the perturbation of the distribution function.

In this paper we extend the result of Ref. [17] by considering an electrostatic drift-kinetic model that accounts for density and temperature background gradients. In this model the coupling between the ion guiding center distribution function and the electrostatic potential is provided by a quasi-neutrality relation which assumes adiabatic electrons and includes polarization effects. The model is a slab version of the system proposed in Ref. [18] to investigate ion temperature gradient driven turbulence in tokamaks.

First, we verify the existence of a Hamiltonian structure for the drift-kinetic model itself. The problem is in principle not trivial due to the presence of the density gradient terms, which impose some restrictions on the boundary conditions, and due to the complexity of 
the quasi-neutrality relation. Then we derive one-moment and two-moment Hamiltonian fluid models starting from the bracket of the drift-kinetic equation expressed in terms of the moments, and looking for a Hamiltonian closure, if necessary. The analysis is carried out for both the full and $\delta f$ orderings, and the resulting fluid models are discussed.

This procedure for deriving Hamiltonian fluid models does not exclude a priori the existence of other Hamiltonian fluid models obtained from the same drift-kinetic equation but adopting different closures. This leaves many open questions concerning the Hamiltonian structure of models obtained from non-Hamiltonian closures.

We remark that the restriction of the analysis to the first two moments with respect to the parallel velocity is of course quite a severe limitation. In particular, it can only lead to very simplified fluid models that evidently cannot account for important physical aspects such as pressure anisotropy, or heat flux evolution. Nevertheless, as it will be seen, it still can lead to reduced models of interest. We foresee the extension to higher order moments, including moments with respect to the magnetic moment coordinate, as a natural extension of the present analysis.

The paper is organized as follows. In Sec. II we present the drift-kinetic model and derive its noncanonical Hamiltonian structure. Sec. III shows how Hamiltonian fluid models can be obtained from the drift-kinetic model, either by sub-algebra arguments, as in the case of the zero moment model, or by determining the Hamiltonian closures, as for the model involving the first two moments. The analysis is carried out first for the full model and in Sec. III C for the $\delta f$ case. Finally, conclusions are drawn in Sec. IV.

\section{THE DRIFT-KINETIC MODEL AND ITS HAMILTONIAN STRUCTURE}

We consider the evolution of a guiding center distribution function $f(x, y, z, v, t)=$ $f_{e q}(x, v)+\delta f(x, y, z, v, t)$ with $x \in[a, b]$ where $a, b \in \mathbb{R}$ and $b>a,(y, z) \in \mathbb{T}^{2}, v \in \mathbb{R}$ and $t \in \mathbb{R}$ with $t \geq 0$, The symbols $x, y$ and $z$ indicate the spatial coordinates, whereas $v$ represents the velocity coordinate in the direction parallel to the magnetic field $\mathbf{B}=B \hat{z}$. 
The latter is assumed to be uniform and constant. The distribution function $f$ has to be considered as the result of an integration with respect to the magnetic moment $\mu$, so that $f(x, y, z, v, t)=\int d \mu f_{\mu}(x, y, z, v, t)$, where $f_{\mu}$ is the actual drift-kinetic distribution function, labelled by the parameter $\mu$.

The equilibrium distribution function $f_{e q}$ contains the information about the equilibrium gradients and can be taken, for instance, to be a Maxwellian

$$
f_{e q}(x, v)=n_{0}(x)\left(M /\left(2 \pi T_{i}(x)\right)\right)^{1 / 2} \exp \left(-M v^{2} /\left(2 T_{i}(x)\right)\right)
$$

as in Ref. [18], where $M$ is the ion mass, whereas $n_{0}(x)$ and $T_{i}(x)$ are some prescribed positive valued functions representing the ion guiding center density and temperature equilibria. Physically interesting cases, for instance for ion temperature gradient driven turbulence, assume radially decreasing profiles, so that $n_{0}(b)<n_{0}(a)$ and $T_{i}(b)<T_{i}(a)$. Our analysis, however, will not depend on the specific expression of $f_{e q}$, provided that this is a function decaying to zero sufficiently rapidly for $v \rightarrow \pm \infty$ and that $T_{i}(x)$ and $n_{0}(x)=\int d v f_{e q}(x, v)$ are positive valued functions. Concerning the perturbation $\delta f$ we also assume that it decays to zero sufficiently rapidly for $v \rightarrow \pm \infty$ but we impose Dirichlet boundary conditions with vanishing data at $x=a$ and $x=b$. Periodicity is assumed along the $y$ and $z$ directions. We consider then the following drift-kinetic equation

$$
\frac{\partial \delta f}{\partial t}+\mathbf{v}_{g c} \cdot \nabla_{\perp} \delta f+v \frac{\partial \delta f}{\partial z}-\frac{q}{M} \frac{\partial \phi}{\partial z} \frac{\partial \delta f}{\partial v}-\frac{c}{B} \frac{\partial \phi}{\partial y} \frac{\partial f_{e q}}{\partial x}-\frac{q}{M} \frac{\partial \phi}{\partial z} \frac{\partial f_{e q}}{\partial v}=0
$$

in which the the electrostatic potential $\phi$ is related to the perturbation of the distribution function $\delta f$ by means of the quasi-neutrality relation

$$
-\nabla_{\perp} \cdot\left[\frac{n_{0}(x)}{B \Omega_{0}} \nabla_{\perp} \phi\right]+\frac{e n_{0}(x)}{T_{e}(x)}(\phi-<\phi>)=\int d v \delta f .
$$

In (3), $\Omega_{0}$ is the ion cyclotron frequency, $e$ is the unit charge, $q$ the charge of the ion species, $<\phi>=(1 / 2 \pi) \int d z \phi$ is an average of the potential along magnetic field lines and $T_{e}(x)$ is the electron temperature profile, which, again is taken to be some prescribed positive valued function. We specify also that $\mathbf{v}_{g c}=c \hat{z} \times \nabla \phi / B$ is the $\mathbf{E} \times \mathbf{B}$ drift, with $c$ equal to the speed of light, whereas the symbol $\nabla_{\perp}$ indicates the gradient along the $x$ and $y$ directions. 
The system (2)-(3) corresponds to a slab version of the drift-kinetic system adopted in Ref. [18], and which has been used to investigate ion temperature gradient turbulence assuming a drift-kinetic description for the ions and an adiabatic response for the electrons.

\section{A. Hamiltonian structure of the drift-kinetic model}

Neglecting the terms depending on $f_{e q}(x, v)$ in Eq. (2), the system (2)-(3) can be shown to possess a Hamiltonian structure [17], if one is able to show that the relation between $\phi$ and $\delta f$ can be written in the form $\mathcal{L} \phi=\int d v \delta f$, where $\mathcal{L}$ is a linear, invertible operator, which is also symmetric with respect to the inner product in $L^{2}(\mathcal{D})$, where $\mathcal{D}$, in our case, is given by $\mathcal{D}=[a, b] \times \mathbb{T}^{2}$.

From (3) we deduce that the operator $\mathcal{L}$ is defined by

$$
\mathcal{L} \phi=-\nabla_{\perp} \cdot\left[\frac{n_{0}(x)}{B \Omega_{0}} \nabla_{\perp} \phi\right]+\frac{e n_{0}(x)}{T_{e}(x)}(\phi-<\phi>)
$$

for $\phi: \mathcal{D} \rightarrow \mathbb{R}$. This operator is evidently linear. Concerning the symmetry property, if we consider two functions $u, w: \mathcal{D} \rightarrow \mathbb{R}$, and suppose that both functions vanish at $x=a$ and $x=b$, we have

$$
\begin{aligned}
& <u \mid \mathcal{L} w>=\int d^{3} x u\left(-\nabla_{\perp} \cdot\left[\frac{n_{0}(x)}{B \Omega_{0}} \nabla_{\perp} w\right]+\frac{e n_{0}(x)}{T_{e}(x)}\left(w-\frac{\int d z^{\prime} w}{2 \pi}\right)\right)= \\
& =\int d^{3} x\left(\frac{n_{0}(x)}{B \Omega_{0}} \nabla_{\perp} u \cdot \nabla_{\perp} w+u \frac{e n_{0}(x)}{T_{e}(x)} w\right)-\int d x d y d z^{\prime} w \frac{e n_{0}(x)}{T_{e}(x)} \frac{\int d z u}{2 \pi}= \\
& =\int d^{3} x\left(-w \nabla_{\perp} \cdot\left[\frac{n_{0}(x)}{B \Omega_{0}} \nabla_{\perp} u\right]+w \frac{e n_{0}(x)}{T_{e}(x)} u\right)-\int d^{3} x w \frac{e n_{0}(x)}{T_{e}(x)} \frac{\int d z^{\prime} u}{2 \pi}= \\
& =<w \mid \mathcal{L} u>
\end{aligned}
$$

where boundary terms vanished when integrating by parts, due to the boundary conditions. Thus, the operator $\mathcal{L}$ is symmetric. With regard to invertibility, one has to show that, for every function $w$ belonging to the image of $\mathcal{L}$, there exists a unique function $u: \mathcal{D} \rightarrow \mathbb{R}$ such that

$$
\mathcal{L} u=w .
$$

Again, $u$ and $w$ are supposed to vanish at $x=a$ and $x=b$. By virtue of this and of the 
periodicity along $y$ and $z$ we can write

$$
\begin{aligned}
u(x, y, z) & =\sum_{k_{y}, k_{z}} u_{k_{y}, k_{z}}(x) \mathrm{e}^{i\left(k_{y} y+k_{z} z\right)}, \\
w(x, y, z) & =\sum_{k_{y}, k_{z}} w_{k_{y}, k_{z}}(x) \mathrm{e}^{i\left(k_{y} y+k_{z} z\right)} .
\end{aligned}
$$

The associated eigenvalue problem $\mathcal{L} u=\lambda u$, with eigenvalue $\lambda$, is then given by

$$
\begin{array}{r}
-\sum_{k_{y}} \mathrm{e}^{i k_{y} y}\left[\left(\frac{n_{0}(x)}{B \Omega_{0}} u_{k_{y}, 0}^{\prime}\right)^{\prime}-\left(k_{y}^{2} n_{0}(x)-\lambda\right) u_{k_{y}, 0}+\right. \\
\left.\sum_{k_{z} \neq 0} \mathrm{e}^{i k_{z} z}\left(\left(\frac{n_{0}(x)}{B \Omega_{0}} u_{k_{y}, k_{z}}^{\prime}\right)^{\prime}-\left(n_{0}(x) \frac{k_{y}^{2}}{B \Omega_{0}}+n_{0}(x) \frac{e}{T_{e}(x)}-\lambda\right) u_{k_{y}, k_{z}}\right)\right]=0,
\end{array}
$$

where the prime denotes derivative with respect to the argument, which is $x$ in this case. This leads, upon introducing the differential operators $\mathcal{L}_{k_{y}, k_{z}}$, to the following set of ordinary differential equations

$$
\begin{array}{r}
\mathcal{L}_{k_{y}, 0} \operatorname{Re} u_{k_{y}, 0}=-\left(\frac{n_{0}(x)}{B \Omega_{0}} \operatorname{Re} u_{k_{y}, 0}^{\prime}\right)^{\prime}+n_{0}(x) k_{y}^{2} \operatorname{Re} u_{k_{y}, 0}=\lambda \operatorname{Re} u_{k_{y}, 0}, \\
\mathcal{L}_{k_{y}, k_{z}} \operatorname{Re} u_{k_{y}, k_{z}}=-\left(\frac{n_{0}(x)}{B \Omega_{0}} \operatorname{Re} u_{k_{y}, k_{z}}^{\prime}\right)^{\prime}+n_{0}(x)\left(\frac{k_{y}^{2}}{B \Omega_{0}}+\frac{e}{T_{e}(x)}\right) \operatorname{Re} u_{k_{y}, k_{z}} \\
=\lambda \operatorname{Re} u_{k_{y}, k_{z}}, \quad \text { for } k_{z} \neq 0
\end{array}
$$

for all $k_{y}$ and $k_{z}$, and similarly for the imaginary part of the Fourier coefficients of $u$. Invertibility amounts to show that the zero eigenvalue does not belong to the spectrum of any operator $\mathcal{L}_{k_{y}, k_{z}}$. We consider first Eq. (9). Upon multiplying both sides times $u_{k_{y}, 0}$ and integrating between $a$ and $b$ we obtain

$$
-\int_{a}^{b} d x\left(\operatorname{Re} u_{k_{y}, 0}\left(\frac{n_{0}(x)}{B \Omega_{0}} \operatorname{Re} u_{k_{y}, 0}^{\prime}\right)^{\prime}-k_{y}^{2} \frac{n_{0}(x)}{B \Omega_{0}}\left(\operatorname{Re} u_{k_{y}, 0}\right)^{2}\right)=\lambda \int_{a}^{b} d x\left(\operatorname{Re} u_{k_{y}, 0}\right)^{2} .
$$

Carrying out an integration by parts we obtain that the eigenvalues satisfy the following relation:

$$
\lambda=\frac{\int_{a}^{b} d x n_{0}(x)\left(\operatorname{Re} u_{k_{y}, 0}^{\prime}\right)^{2}+k_{y}^{2} \int_{a}^{b} d x n_{0}(x)\left(\operatorname{Re} u_{k_{y}, 0}\right)^{2}}{B \Omega_{0} \int_{a}^{b} d x\left(\operatorname{Re} u_{k_{y}, 0}\right)^{2}} .
$$

Because $n_{0}(x)$ takes only positive values, it follows from (12), that all the eigenvalues are positive, and in particular that 0 cannot be an eigenvalue. 
The same procedure, applied to Eq. (10), leads to

$$
\lambda=\frac{\int_{a}^{b} d x n_{0}(x)\left(\operatorname{Re} u_{k_{y}, 0}^{\prime}\right)^{2}+\int_{a}^{b} d x n_{0}(x)\left(k_{y}^{2}+B \Omega_{0} \frac{e}{T_{e}(x)}\right)\left(\operatorname{Re} u_{k_{y}, 0}\right)^{2}}{B \Omega_{0} \int_{a}^{b} d x\left(\operatorname{Re} u_{k_{y}, 0}^{2}\right)^{2}} .
$$

Similarly, because also $T_{e}(x)$ is a positive valued function, 0 is not an eigenvalue. Thus, for each $k_{y}$ and $k_{z}$, the operators $\mathcal{L}_{k_{y}, k_{z}}$ are invertible. In particular, one can invert all of the following relations

$$
\begin{array}{r}
\mathcal{L}_{0,0} u_{0,0}=w_{0,0}, \\
\mathcal{L}_{k_{y}, 0} u_{k_{y}, 0}=w_{k_{y}, 0}, \quad \text { for } k_{y} \neq 0 \\
\mathcal{L}_{k_{y}, k_{z}} u_{k_{y}, k_{z}}=w_{k_{y}, k_{z}}, \quad \text { for } k_{y} \neq 0, k_{z} \neq 0 .
\end{array}
$$

and consequently reconstruct the inverse operator

$$
u=\mathcal{L}^{-1} w=\sum_{k_{y}, k_{z}} \mathcal{L}_{k_{y}, k_{z}}^{-1} w_{k_{y}, k_{z}} \mathrm{e}^{i\left(k_{y} y+k_{z} z\right)}
$$

Denoting $L=\mathcal{L}^{-1}$, we can then write $\phi=L \int d v \delta f$.

For $f_{e q}=0$, the system can then be written in the Hamiltonian form

$$
\frac{\partial \delta f}{\partial t}=\{\delta f, H\}
$$

with Hamiltonian functional given by

$$
H(\delta f)=\int d^{3} x d v \delta f\left(M \frac{v^{2}}{2}+q \frac{L}{2} \int d v^{\prime} \delta f\left(\mathbf{x}, v^{\prime}\right)\right)
$$

and Poisson bracket of noncanonical type corresponding to

$$
\{F, G\}=\int d^{3} x d v \delta f\left(\left[F_{\delta f}, G_{\delta f}\right]_{x}+\left[F_{\delta f}, G_{\delta f}\right]_{v}\right)
$$

where

$$
[f, g]_{x}=-\frac{c}{q B}\left(\frac{\partial f}{\partial x} \frac{\partial g}{\partial y}-\frac{\partial f}{\partial y} \frac{\partial g}{\partial x}\right), \quad[f, g]_{v}=\frac{1}{M}\left(\frac{\partial f}{\partial z} \frac{\partial g}{\partial v}-\frac{\partial f}{\partial v} \frac{\partial g}{\partial z}\right)
$$

In (20), subscripts on $F$ and $G$ indicate functional derivatives. Note that a linear combination of "inner" brackets $[,]_{x}$ and $[,]_{v}$ corresponds to the bracket yielding the Hamiltonian 
dynamics of the guiding center at the particle level. The corresponding Hamiltonian field equation for $\delta f$, has been obtained by "lifting" the dynamics at the particle level, to the field level (for a general theory of "lifting" Hamiltonian dynamics, see Refs. [19, 20]).

The terms in (2) depending on the equilibrium distribution function can be reintegrated in the system by considering the following modified version of the Poisson bracket:

$$
\{F, G\}=\int d^{3} x d v\left(f_{e q}+\delta f\right)\left(\left[F_{\delta f}, G_{\delta f}\right]_{x}+\left[F_{\delta f}, G_{\delta f}\right]_{v}\right) .
$$

In order to see that the operation (22) is indeed a Poisson bracket, and in particular that it satisfies the Jacobi identity (bilinearity, Leibniz identity and antisymmetry are obvious), we first investigate under what conditions (22) can be written in the form

$$
\{F, G\}=\int d^{3} x d v F_{\delta f} J(\delta f) G_{\delta f}
$$

where $J$ is the cosymplectic operator, depending on $\delta f$, and which is required to be anti symmetric. The bilinear form (22) can indeed be cast in the form (23), provided that boundary terms vanish when integrating by parts. Because in general $f_{e q}(a, v) \neq f_{e q}(b, v)$, this occurs if the functional derivatives $F_{\delta f}$ and $G_{\delta f}$ vanish at $x=a$ and $x=b$, or if they do not depend on the coordinates $x$ and $y$. Here and in the following, we will then assume that we deal with functionals such that their functional derivatives satisfy one these properties (in addition to the periodicity along $y$ and $z$ ), so that boundary terms resulting from integration by parts are automatically zero. In order to see explicitly how this occurs, we observe that integration by parts yields

$$
\begin{aligned}
& \int d^{3} x d v\left(f_{e q}+\delta f\right)\left[F_{\delta f}, G_{\delta f}\right]_{x} \\
& =-\left.(c / q B) \int d y d z d v\left(f_{e q}+\delta f\right) F_{\delta f} \partial_{y} G_{\delta f}\right|_{a} ^{b}+(c / q B) \int d^{3} x d v F_{\delta f} \partial_{x}\left(\left(f_{e q}+\delta f\right) \partial_{y} G_{\delta f}\right) \\
& +\left.(c / q B) \int d x d z d v\left(f_{e q}+\delta f\right) F_{\delta f} \partial_{x} G_{\delta f}\right|_{\mathbb{T}}-(c / q B) \int d^{3} x d v F_{\delta f} \partial_{y}\left(\left(f_{e q}+\delta f\right) \partial_{x} G_{\delta f}\right)
\end{aligned}
$$

In general, the boundary term arising from the evaluation at $x=a$ and $x=b$ would be finite for $f_{e q}(a, v) \neq f_{e q}(b, v)$. It does, however, vanish if $F_{\delta f}(a, y, z, v)=F_{\delta f}(b, y, z, v)=0$ as we assumed. The boundary term originating from the integration with respect to $y$ vanishes because of the periodicity assumption. If $F_{\delta f}\left(\right.$ or $\left.G_{\delta f}\right)$ does not depend on $x$ and 
$y$, then the only finite contribution to $\{F, G\}$ in Eq. (22) is the one including $\left[F_{\delta f}, G_{\delta f}\right]_{v}$, for which, however, boundary terms vanish automatically because of the periodicity along $z$ and because $f_{e q} \rightarrow 0$ for $v \rightarrow \pm \infty$. With these assumptions, (22) can be written in the form (23) with cosymplectic operator given by $J(\delta f)=-\left[f_{e q}+\delta f, .\right]_{x}-\left[f_{e q}+\delta f, .\right]_{v}$. From this expression one can see that the addition of the contribution depending on $f_{e q}$ does not modify the dependence of $J$ on the dynamical variable $\delta f$. By virtue of the condition on the Jacobi identity shown in Ref. [21], one can then conclude that, because (20) is a Poisson bracket, then (22) is a Poisson bracket too, provided that the above restriction to the involved class of functionals is imposed.

It is then straightforward to see that the Hamiltonian (19) and the Poisson bracket (22), supplemented by the relation (3) yield namely Eq. (2). Note that the functional derivative $H_{\delta f}=M v^{2} / 2+q \phi$ satisfies the required boundary conditions.

\section{HAMILTONIAN CLOSURES FOR THE REDUCED FLUID MODELS}

We consider now the moments of the perturbation of the distribution function, defined as

$$
\tilde{P}_{n}(\mathbf{x})=\int d v v^{n} \delta f(\mathbf{x}, v)
$$

where $n$ is a non-negative integer, and analogously, the moments of the equilibrium distribution function, which we denote with

$$
\bar{P}_{n}(x)=\int d v v^{n} f_{e q}(x, v) .
$$

We intend to see how the Poisson bracket (22) transforms when expressed in terms of the moments (25), which become the new dynamical variables, and of the equilibrium moments (26). Subsequently, we will investigate under what conditions the resulting expression can

still be a Poisson bracket, when restricting to functionals of the first moment only, and of the first two moments. 
The change of variables $\delta f \rightarrow\left\{\tilde{P}_{n}\right\}_{n \in \mathbb{N}}$ induces the following transformation between functional derivatives:

$$
F_{\delta f}=\sum_{n \in \mathbb{N}} v^{n} \bar{F}_{n}
$$

between two functionals $F$ and $\bar{F}$ related by $F(\delta f)=\bar{F}\left(\tilde{P}_{0}, \tilde{P}_{1}, \cdots\right)$. In Eq. (27) we indicated with the subscript $n$ the functional derivative with respect to $\tilde{P}_{n}$. Note that, from (27) the functional derivatives $\bar{F}_{n}$ inherit, from the assumptions on $F_{\delta f}$, the boundary conditions that make boundary terms vanish when integrating by parts with respect to the spatial variables.

Making use of (25), (26) and (27), the Poisson bracket (22) transforms into

$$
\{\bar{F}, \bar{G}\}=\sum_{m, n \in \mathbb{N}} \int d^{3} x\left[\left(\bar{P}_{m+n}+\tilde{P}_{m+n}\right)\left[\bar{F}_{m}, \bar{G}_{n}\right]_{x}+\frac{\bar{P}_{m+n-1}+\tilde{P}_{m+n-1}}{M}\left(n \bar{G}_{n} \partial_{z} \bar{F}_{m}-m \bar{F}_{m} \partial_{z} \bar{G}_{n}\right)\right]
$$

For simplicity, bars indicating the functionals in terms of the moments, will be dropped in the sequel.

\section{A. Functionals of $\tilde{P}_{0}$}

We consider now, as set of observables, the functionals of $\tilde{P}_{0}$ only. The expression for the bracket (28) then reads

$$
\{F, G\}=\int d^{3} x\left(\bar{P}_{0}+\tilde{P}_{0}\right)\left[F_{0}, G_{0}\right]_{x}
$$

The bracket (29) is again a Poisson bracket and in particular it satisfies the Jacobi identity. This can be verified considering that for $\bar{P}_{0}=0$ it has a Lie-Poisson structure [22, 23], and that, as above mentioned, the inclusion of a prescribed function such as $\bar{P}_{0}$, does not alter the dependence of the co-symplectic operator on the dynamical variables. In particular, $\{F, G\}$ is a functional that does not depend on moments other than $\tilde{P}_{0}$. Thus, the set of functionals of $\tilde{P}_{0}$ forms a sub-algebra with respect to the Poisson bracket (28). In this case, the system is automatically Hamiltonian and no need for Hamiltonian closures is required.

The dynamics is generated by a Hamiltonian $H\left(\tilde{P}_{0}\right)$ and the corresponding fluid model is 
given by

$$
\frac{\partial \tilde{P}_{0}}{\partial t}=\left[H_{0}, \bar{P}_{0}+\tilde{P}_{0}\right]_{x}
$$

We compare now Eq. (30) with the equation obtained by taking the zero order moment of Eq. (2) and which reads

$$
\frac{\partial \tilde{P}_{0}}{\partial t}=q\left[L \tilde{P}_{0}, \bar{P}_{0}+\tilde{P}_{0}\right]_{x}-\partial_{z} \tilde{P}_{1}
$$

The term on the right-hand side of Eq. (30) matches the $\mathbf{E} \times \mathbf{B}$ advection term in Eq. (31) if the Hamiltonian is given by

$$
H\left(\tilde{P}_{0}\right)=\int d^{3} x\left[\frac{q}{2} \tilde{P}_{0} L \tilde{P}_{0}+v\left(\bar{P}_{0}+\tilde{P}_{0}\right)\right]
$$

where $v$ is an arbitrary function. Actually, the set of functionals

$$
C\left(\tilde{P}_{0}\right)=\int d^{3} x v\left(\bar{P}_{0}+\tilde{P}_{0}\right)
$$

corresponds to the set of Casimir invariants for the bracket (29), i.e. functionals that commute, through the operation (29), with any other functional of $\tilde{P}_{0}$. The knowledge of such invariants can be useful in principle not only because it provides a quantity preserved by the dynamics, but also for its application in determining equilibria and stability of the system (see, e.g. Ref. [23]). The presence of such functionals in the Hamiltonian does not give any contribution to the equations of motion, and so in principle they can be omitted from Eq. (32). Concerning the second term on the right-hand side of Eq. (31) it is evident that, if we consider closures of the form $\tilde{P}_{1}=\mathcal{E}\left(\tilde{P}_{0}\right)$ for some function $\mathcal{E}$ not involving operators (which is the case for analysis carried out in the whole paper), Eq. (30) matches Eq. (31) if and only if $\mathcal{E}$ is constant or $\tilde{P}_{0}$ does not depend on $z$. In particular, we can consider namely the latter two-dimensional (2D) case and assume a weakly varying density and electron temperature equilibria: $\bar{P}_{0}=n_{0}(\epsilon x) \approx N_{0}+\epsilon N_{0}^{\prime} x$, with $\epsilon \ll 1, N_{0}=n_{0}(0)$, $N_{0}^{\prime}=n_{0}^{\prime}(0)$ and $T_{e}(\epsilon x) \approx T_{e 0}=T_{e}(0)$. We can then consider the following modified version of the quasi-neutrality relation (3):

$$
-\frac{N_{0}}{B \Omega_{0}} \Delta_{\perp} \phi+\frac{e N_{0}}{T_{e 0}} \phi=\int d v \delta f=\tilde{P}_{0}
$$


where the spatial coordinates are now restricted to the domain $\{(x, y): x \in[a, b], y \in \mathbb{T}\}$. The quasi-neutrality relation, which now involves a Helmholtz operator, can still be inverted and written in the form $\phi=L_{H} \tilde{P}_{0}$, with $L_{H}$ the inverse of the corresponding Helmholtz operator. From (29) and (32), we obtain the following expressions for the 2D Poisson bracket and Hamiltonian, up to the contributions due to the Casimirs:

$$
\begin{gathered}
\{F, G\}=\int d^{2} x\left(\epsilon N_{0}^{\prime} x+\tilde{P}_{0}\right)\left[F_{0}, G_{0}\right]_{x} . \\
H\left(\tilde{P}_{0}\right)=\frac{q}{2} \int d^{2} x \tilde{P}_{0} L_{H} \tilde{P}_{0} .
\end{gathered}
$$

Note that the projection to 2D implied an integration over the ignorable coordinate $z$ on a finite domain. This amounts to the multiplication times a constant factor which can however be removed by rescaling, preserving the properties of the Poisson bracket.

Making use of Eq. (34), the equation of motion resulting from (35)-(36) reads

$$
\frac{\partial}{\partial t}\left(\frac{N_{0}}{B \Omega_{0}} \Delta_{\perp} \phi-\frac{e N_{0}}{T_{e 0}} \phi\right)-q\left[\phi, \frac{N_{0}}{B \Omega_{0}} \Delta_{\perp} \phi\right]_{x}+\epsilon N_{0}^{\prime} \frac{c}{B} \frac{\partial \phi}{\partial y}=0 .
$$

Eq. (37) corresponds to the well known Charney-Hasegawa-Mima equation [24, 25], which describes the dynamics of Rossby waves in planetary atmospheres and of drift waves in plasmas. With Eqs. (35)-(36) we recovered the Hamiltonian structure of the CharneyHasegawa-Mima equation, whose Poisson bracket is then shown to emerge from the bracket (28), when restricting to functionals of the zero order moment only. The Hamiltonian structure (35)-(36) was discovered in Ref. [26] and recently rederived in the context of procedures for obtaining reduced fluid models preserving the Hamiltonian structure of a parent model $[27,28]$. We finally note that the choice of retaining the linear term of the development of $n_{0}$ in the bracket (35) and not in the quasi-neutrality relation (34), has been made to obey, in the final equation of motion, the ordering underlying the CharneyHasegawa-Mima equation. Indeed, such equation assumes that also the density and potential fluctuations (corresponding to $\tilde{P}_{0} / N_{0}$ and $e \phi / T_{e 0}$ in our system, respectively) be both of order $\epsilon$. Therefore, expanding further $n_{0}$ in Eq. (34) leads to terms that should be neglected. We notice also that, according to the ordering of the Charney-Hasegawa-Mima equation, the 
(normalized) time derivative operator $\partial_{t}$ is also supposed to be of order $\epsilon$, expressing the fact that the equation refers to low frequency fluctuations. Each term of the CharneyHasegawa-Mima equation, is then of order $\epsilon^{2}$. In the Hamiltonian structure, this reflects in the cosymplectic operator of the Poisson bracket (35) and in the functional derivatives of the Hamiltonian (36) both being of order $\epsilon$.

\section{B. Functionals of $\tilde{P}_{0}$ and $\tilde{P}_{1}$}

We restrict now to functionals $F$ and $G$ depending only on the first two moments $\tilde{P}_{0}$ and $\tilde{P}_{1}$. With the above restrictions the Poisson bracket (28) reduces to

$$
\begin{aligned}
& \{F, G\}=\int d^{3} x\left[\left(\bar{P}_{0}+\tilde{P}_{0}\right)\left[F_{0}, G_{0}\right]_{x}+\left(\bar{P}_{1}+\tilde{P}_{1}\right)\left(\left[F_{1}, G_{0}\right]_{x}+\left[F_{0}, G_{1}\right]_{x}\right)+\left(\bar{P}_{2}+\tilde{P}_{2}\right)\left[F_{1}, G_{1}\right]_{x}\right. \\
& \left.+\frac{\bar{P}_{0}+\tilde{P}_{0}}{M}\left(G_{1} \partial_{z} F_{0}-F_{1} \partial_{z} G_{0}\right)+\frac{\bar{P}_{1}+\tilde{P}_{1}}{M}\left(G_{1} \partial_{z} F_{1}-F_{1} \partial_{z} G_{1}\right)\right] .
\end{aligned}
$$

The bilinear form (38) is not guaranteed to be a Poisson bracket. Indeed, such expression

depends explicitly on $\tilde{P}_{2}$, which shows that, unlike the case of Vlasov equation $[14,16]$, the set of functionals of the first two moments does not form a sub-algebra of the algebra of functionals of all the moments. In particular, the Jacobi identity is not respected by (38). We suppose then that a closure relation $\tilde{P}_{2}=\tilde{\mathcal{F}}\left(\tilde{P}_{0}, \tilde{P}_{1}\right)$ exists (where we assume that the function $\tilde{\mathcal{F}}$ does not involve integral or differential operator, or explicit dependence on the coordinates) and look for Hamiltonian closures, that is functions $\tilde{\mathcal{F}}$ such that, when inserted into (38) yield a Poisson bracket.

We note, however, that by means of the change of variables

$$
P_{n}=\bar{P}_{n}+\tilde{P}_{n}, \quad n \in \mathbb{N}
$$

the form (38) becomes

$$
\begin{aligned}
& \{F, G\}=\int d^{3} x\left[P_{0}\left[F_{0}, G_{0}\right]_{x}+P_{1}\left(\left[F_{1}, G_{0}\right]_{x}+\left[F_{0}, G_{1}\right]_{x}\right)+P_{2}\left[F_{1}, G_{1}\right]_{x}\right. \\
& \left.+\frac{P_{0}}{M}\left(G_{1} \partial_{z} F_{0}-F_{1} \partial_{z} G_{0}\right)+\frac{P_{1}}{M}\left(G_{1} \partial_{z} F_{1}-F_{1} \partial_{z} G_{1}\right)\right]
\end{aligned}
$$


where the subscripts on the functionals $F$ and $G$ now indicate functional derivatives with respect to $P_{n}$. The form (40) is now identical to that examined in Ref. [17] and we can take advantage of the corresponding results. In particular, we remark that under the change of variables (39), the functional derivatives are related, for each $n$, by $\delta \bar{F} / \delta \tilde{P}_{n}=\delta F / \delta P_{n}$, where $\bar{F}\left(\tilde{P}_{0}, \tilde{P}_{1}, \cdots\right)=F\left(P_{0}, P_{1}, \cdots\right)$. Consequently, the functional derivatives with respect to $P_{n}$ satisfy the same boundary conditions as those with respect to $\tilde{P}_{n}$, which in particular make boundary terms vanish when integrating by parts. Thus, in spite of the fact that the moments $P_{n}$ do not necessarily satisfy the same boundary conditions of the moments adopted in Ref. [17], the same procedure for determining the Hamiltonian closures can be applied. Such procedure is described in detail in Ref. [17] and consequently we report here only its main steps.

First, in the bracket $(40)$ we replace $P_{2}$ with $\mathcal{F}\left(P_{0}, P_{1}\right)\left(\right.$ where $\left.\mathcal{F}\left(P_{0}, P_{1}\right)=\bar{P}_{2}+\tilde{\mathcal{F}}_{(}\left(\tilde{P}_{0}, \tilde{P}_{1}\right)\right)$. The bracket (40) is then decomposed as the sum of two contributions:

$$
\{F, G\}=\{F, G\}_{c}+\{F, G\}_{h}
$$

where

$$
\begin{array}{r}
\{F, G\}_{c}=\int d^{3} x\left[P_{0}\left[F_{0}, G_{0}\right]_{x}+P_{1}\left(\left[F_{1}, G_{0}\right]_{x}+\left[F_{0}, G_{1}\right]_{x}\right)+\right. \\
\left.+\frac{P_{0}}{M}\left(G_{1} \partial_{z} F_{0}-F_{1} \partial_{z} G_{0}\right)+\frac{P_{1}}{M}\left(G_{1} \partial_{z} F_{1}-F_{1} \partial_{z} G_{1}\right)\right] \\
\{F, G\}_{h}=\int d^{3} x \mathcal{F}\left[F_{1}, G_{1}\right]_{x}
\end{array}
$$

The Jacobi identity for (41) then reads as

$$
\begin{aligned}
& \left\{\{F, G\}_{c}, H\right\}_{c}+\left\{\{F, G\}_{c}, H\right\}_{h} \\
& +\left\{\{F, G\}_{h}, H\right\}_{c}+\left\{\{F, G\}_{h}, H\right\}_{h}+\circlearrowleft=0, \quad \forall F, G, H,
\end{aligned}
$$

where $\circlearrowleft$ indicates the additional terms obtained by cyclic permutations. The bracket $\{,\}_{c}$ is then split further into two contributions:

$$
\{F, G\}_{c}=\{F, G\}_{x}+\{F, G\}_{V}
$$


where

$$
\begin{array}{r}
\{F, G\}_{x}=\int d^{3} x\left[P_{0}\left[F_{0}, G_{0}\right]_{x}+P_{1}\left(\left[F_{1}, G_{0}\right]_{x}+\left[F_{0}, G_{1}\right]_{x}\right)\right] \\
\{F, G\}_{V}=\int d^{3} x\left[\frac{P_{0}}{M}\left(G_{1} \partial_{z} F_{0}-F_{1} \partial_{z} G_{0}\right)+\frac{P_{1}}{M}\left(G_{1} \partial_{z} F_{1}-F_{1} \partial_{z} G_{1}\right)\right] .
\end{array}
$$

Taking advantage from the fact that $\{,\}_{x}$ and $\{,\}_{V}$ independently satisfy the Jacobi identity, we can conclude that

$$
\left\{\{F, G\}_{c}, H\right\}_{c}+\circlearrowleft=\left\{\{F, G\}_{x}, H\right\}_{V}+\left\{\{F, G\}_{V}, H\right\}_{x}+\circlearrowleft
$$

With the help of the above mentioned result of Ref. [21], direct calculations lead to

$$
\left\{\{F, G\}_{c}, H\right\}_{c}+\circlearrowleft=2 \int d^{3} x \frac{P_{1}}{M} \partial_{z} F_{0}\left[G_{1}, H_{1}\right]_{x}+\circlearrowleft
$$

The remaining contributions in (42), on the other hand, turn out to correspond to

$$
\begin{aligned}
& \left\{\{F, G\}_{h}, H\right\}_{h}+\circlearrowleft=\int d^{3} x \mathcal{F}\left[F_{1}, G_{1}\right]_{x}\left[\partial_{1} \mathcal{F}, H_{1}\right]_{x}+\circlearrowleft, \\
& \left\{\{F, G\}_{c}, H\right\}_{h}+\circlearrowleft=\int d^{3} x \mathcal{F}\left[\left[F_{1}, G_{0}\right]_{x}+\left[F_{0}, G_{1}\right]_{x}\right. \\
& \left.+\left(G_{1} / M\right) \partial_{z} F_{1}-\left(F_{1} / M\right) \partial_{z} G_{1}, H_{1}\right]_{x}+\circlearrowleft, \\
& \left\{\{F, G\}_{h}, H\right\}_{c}+\circlearrowleft \\
& =\int d^{3} x\left[P_{0}\left[\partial_{0} \mathcal{F}\left[F_{1}, G_{1}\right]_{x}, H_{0}\right]_{x}+P_{1}\left(\left[\partial_{1} \mathcal{F}\left[F_{1}, G_{1}\right]_{x}, H_{0}\right]_{x}+\left[\partial_{0} \mathcal{F}\left[F_{1}, G_{1}\right]_{x}, H_{1}\right]_{x}\right)\right. \\
& +\left(P_{1} / M\right)\left(H_{1} \partial_{z}\left(\partial_{1} \mathcal{F}\left[F_{1}, G_{1}\right]_{x}\right)-\partial_{1} \mathcal{F}\left[F_{1}, G_{1}\right]_{x} \partial_{z} H_{1}\right) \\
& \left.+\left(P_{0} / M\right)\left(H_{1} \partial_{z}\left(\partial_{0} \mathcal{F}\left[F_{1}, G_{1}\right]_{x}\right)-\partial_{1} \mathcal{F}\left[F_{1}, G_{1}\right]_{x} \partial_{z} H_{0}\right)\right]+\circlearrowleft .
\end{aligned}
$$

In (48) we indicated with $\partial_{0}$ and $\partial_{1}$ the partial derivatives with respect to $P_{0}$ and $P_{1}$.

The contributions from $\left\{\{F, G\}_{c}, H\right\}_{c}$ and from $\left\{\{F, G\}_{h}, H\right\}_{c}$ are the only ones that possess terms that contain $\partial_{z} H_{0}\left[F_{1}, G_{1}\right]_{x}$. Such terms then need to cancel each other, which occurs, for arbitrary $F, G$ and $H$, if and only if $\partial_{1} \mathcal{F}=2 P_{1}$. This implies

$$
\mathcal{F}\left(P_{0}, P_{1}\right)=\frac{P_{1}^{2}}{P_{0}}+\theta\left(P_{0}\right)
$$

with $\theta$ arbitrary function. Inserting the form (49) into Eq. (42) one obtains first that all the contributions not involving $z$-derivatives vanish for any $\theta$. The remaining contributions 
imply

$$
\int d^{3} x\left(3 \theta-P_{0} \theta^{\prime}\right)\left[F_{1}, G_{1}\right]_{x} \partial_{z} H_{1}+\circlearrowleft=0,
$$

for all $F, G$ and $H$. This eventually leads to

$$
\mathcal{F}\left(P_{0}, P_{1}\right)=\frac{P_{1}^{2}}{P_{0}}+\mathcal{A} P_{0}^{3}
$$

where $\mathcal{A}$ is an arbitrary constant. Given that $P_{n}=\int d v v^{n}\left(f_{e q}+\delta f\right)$, this amounts to say that the only Hamiltonian closure for the first two moments of the ion temperature gradient drift-kinetic model (2)-(3) corresponds to a plasma for which the pressure $\mathbb{P}=P_{2}-P_{1}^{2} / P_{0}$, referred to the total distribution function $f_{e q}+\delta f$, is equal to $\mathcal{A} P_{0}^{3}$. This corresponds to the adiabatic equation of state for an ideal gas composed of molecules with one degree of freedom. For $\mathcal{A}=0$ one retrieves the cold plasma closure.

Going back to the variables $\tilde{P}_{0}$ and $\tilde{P}_{1}$, we obtain that the resulting Poisson bracket is given by

$$
\begin{aligned}
& \{F, G\}=\int d^{3} x\left[\left(\bar{P}_{0}+\tilde{P}_{0}\right)\left[F_{0}, G_{0}\right]_{x}+\left(\bar{P}_{1}+\tilde{P}_{1}\right)\left(\left[F_{1}, G_{0}\right]_{x}+\left[F_{0}, G_{1}\right]_{x}\right)\right. \\
& +\left(\frac{\left(\bar{P}_{1}+\tilde{P}_{1}\right)^{2}}{\bar{P}_{0}+\tilde{P}_{0}}+\mathcal{A}\left(\bar{P}_{0}+\tilde{P}_{0}\right)^{3}\right)\left[F_{1}, G_{1}\right]_{x}+\frac{\bar{P}_{0}+\tilde{P}_{0}}{M}\left(G_{1} \partial_{z} F_{0}-F_{1} \partial_{z} G_{0}\right) \\
& \left.+\frac{\bar{P}_{1}+\tilde{P}_{1}}{M}\left(G_{1} \partial_{z} F_{1}-F_{1} \partial_{z} G_{1}\right)\right] .
\end{aligned}
$$

Considering a generic Hamiltonian functional $H\left(\tilde{P}_{0}, \tilde{P}_{1}\right)$, we obtain that the dynamical equations generated by (52) and $H$ are given by

$$
\begin{gathered}
\frac{\partial \tilde{P}_{0}}{\partial t}=\left[H_{0}, \bar{P}_{0}+\tilde{P}_{0}\right]_{x}+\left[H_{1}, \bar{P}_{1}+\tilde{P}_{1}\right]_{x}-\frac{\partial_{z}\left(\left(\bar{P}_{0}+\tilde{P}_{0}\right) H_{1}\right)}{M} \\
\frac{\partial \tilde{P}_{1}}{\partial t}=\left[H_{0}, \bar{P}_{1}+\tilde{P}_{1}\right]_{x}+\left[H_{1} \frac{\left(\bar{P}_{1}+\tilde{P}_{1}\right)^{2}}{\bar{P}_{0}+\tilde{P}_{0}}+\mathcal{A}\left(\bar{P}_{0}+\tilde{P}_{0}\right)^{3}\right]_{x}-\frac{\bar{P}_{0}+\tilde{P}_{0}}{M} \partial_{z} H_{0}-\frac{\partial_{z}\left(\left(\bar{P}_{1}+\tilde{P}_{1}\right) H_{1}\right)}{M} \\
-\frac{\bar{P}_{1}+\tilde{P}_{1}}{M} \partial_{z} H_{1}
\end{gathered}
$$

whereas the first two moments of the drift-kinetic equation (2) yield

$$
\begin{array}{r}
\frac{\partial \tilde{P}_{0}}{\partial t}=q\left[L \tilde{P}_{0}, \bar{P}_{0}+\tilde{P}_{0}\right]_{x}-\partial_{z} \tilde{P}_{1} \\
\frac{\partial \tilde{P}_{1}}{\partial t}=-q\left[L \tilde{P}_{0}, \bar{P}_{1}+\tilde{P}_{1}\right]_{x}-\partial_{z} \tilde{P}_{2}-\frac{q}{M}\left(\bar{P}_{0}+\tilde{P}_{0}\right) \partial_{z} L \tilde{P}_{0}
\end{array}
$$


Eqs. (53)-(54) match Eqs. (55)-(56) if the above Hamiltonian closure $\tilde{P}_{2}=-\bar{P}_{2}+\left(\tilde{P}_{1}+\right.$ $\left.\bar{P}_{1}\right)^{2} /\left(\tilde{P}_{0}+\bar{P}_{0}\right)+\mathcal{A}\left(\bar{P}_{0}+\tilde{P}_{0}\right)^{3}$ is adopted and if the Hamiltonian is given by

$$
H\left(\tilde{P}_{0}, \tilde{P}_{1}\right)=\int d^{3} x\left(\frac{1}{2} \frac{\left(\bar{P}_{1}+\tilde{P}_{1}\right)^{2}}{\bar{P}_{0}+\tilde{P}_{0}}+\frac{q}{2} \tilde{P}_{0} L \tilde{P}_{0}+\mathcal{A}\left(\bar{P}_{0}+\tilde{P}_{0}\right)^{3}\right),
$$

which amounts to expressing the Hamiltonian (19) in terms of the first three moments and then imposing the Hamiltonian closure. Note that the equilibrium second order moment $\bar{P}_{2}$, due to its independence on $z$, is absent from the equations of motion. It is also absent from the expression of the Hamiltonian (57) because, after imposing the closure in the Hamiltonian, its contribution would amount to a constant term, which is irrelevant for the dynamics.

If we specialize to the case $f_{e q}=n_{0}(x)\left(M /\left(2 \pi T_{i}(x)\right)\right)^{1 / 2} \exp \left(-M v^{2} /\left(2 T_{i}(x)\right)\right)$, which implies $\bar{P}_{0}=n_{0}(x), \bar{P}_{1}=0$ and $\bar{P}_{2}=\frac{T_{i}(x)}{M} n_{0}(x)$, the resulting system of fluid equations (55)-(56) can be written as

$$
\begin{aligned}
\frac{\partial \tilde{n}}{\partial t}-q[\phi, \tilde{n}]_{x}-\frac{c}{B} n_{0}^{\prime} \frac{\partial \phi}{\partial y}+\frac{\partial\left[\left(n_{0}+\tilde{n}\right) \tilde{u}\right]}{\partial z} & =0, \\
\frac{\partial \tilde{u}}{\partial t}-q[\phi, \tilde{u}]_{x}+\frac{1}{M} \frac{\partial}{\partial z}\left(M \frac{\tilde{u}^{2}}{2}+q \varphi+\frac{3}{2} \mathcal{A}\left(n_{0}+\tilde{n}\right)^{2}\right) & =0,
\end{aligned}
$$

where we introduced $\tilde{n}=\tilde{P}_{0}$ and $\tilde{u}=\tilde{P}_{1} /\left(n_{0}+\tilde{P}_{0}\right)$, which correspond to the density and parallel velocity fluctuations, respectively. Eqs. (58)-(59) provide then a two-moment model, which is Hamiltonian by construction and which conserves the total energy

$$
H(\tilde{n}, \tilde{u})=\int d^{3} x\left[M\left(n_{0}+\tilde{n}\right) \frac{\tilde{u}^{2}}{2}+q \tilde{n} L \tilde{n}+\frac{\mathcal{A}}{2}\left(n_{0}+\tilde{n}\right)^{3}\right] .
$$

Making use of the quasi-neutrality relation (3), the system (58)-(59) can then be seen as a generalized 3D Charney-Hasegawa-Mima equation, coupled with an evolution equation for the parallel velocity. The fluid system depends on the density profile $n_{0}$ and on the electron temperature profile $T_{e}$ (through the quasi-neutrality relation), but not on the ion temperature profile $T_{i}$, because, as specified above, adopting the Hamiltonian closure makes the equations of motion independent on $\bar{P}_{2}$, and consequently on $T_{i}$. Higher order moments should be invoked in order to have an influence of the ion temperature profile on the fluid equations. 
In the limit $n_{0}=0$, the model (58)-(59) corresponds to the fluid model derived in Ref. [32] by taking moments of the drift-kinetic equation for a mono water-bag distribution function. This model turns out then to have a noncanonical Hamiltonian structure.

\section{Hamiltonian models obtained from the $\delta f$ ordering}

It is also interesting to apply to the drift-kinetic equation (2) the so called $\delta f$ ordering (see, e.g. Ref. [29]), which assumes low frequencies, small fluctuations and weak variations along the guide field. If we also assume a Maxwellian equilibrium distribu-

tion function, with weak spatial variations, given by $f_{e q}(v)=\left(N_{0}+\epsilon N_{0}^{\prime} x\right) F_{M}(v)$, where $F_{M}(v)=\left(M /\left(2 \pi T_{i 0}\right)\right)^{1 / 2} \exp \left(-M v^{2} /\left(2 T_{i 0}\right)\right)$ and $T_{i 0}=T_{i}(0)$, we obtain

$$
\frac{\partial \delta f}{\partial t}+\mathbf{v}_{g c} \cdot \nabla_{\perp} \delta f+v \frac{\partial \delta f}{\partial z}-\frac{c}{B} \epsilon N_{0}^{\prime} F_{M} \frac{\partial \phi}{\partial y}-\frac{q}{M} N_{0} \frac{\partial \phi}{\partial z} F_{M}^{\prime}=0 .
$$

In (61) only terms of the order of $\epsilon^{2}$ have been retained, after having assumed $\tau \partial_{t} \sim \delta f / f_{e q} \sim$ $\partial_{z} / \partial_{x} \sim \partial_{z} / \partial_{y} \sim \epsilon \ll 1$, where $\tau$ is a characteristic time. We also assume that $\phi$ is related to $\delta f$ by means of the quasi-neutrality relation (34).

It can be verified directly that the system (61), complemented by (34), possesses a Hamiltonian structure given by

$$
\begin{gathered}
\{F, G\}=\int d^{3} x d v\left[\left(\delta f+\epsilon N_{0}^{\prime} x F_{M}\right)\left[F_{\delta f}, G_{\delta f}\right]_{x}+\frac{N_{0}}{M} F_{M}^{\prime} F_{\delta f} \partial_{z} G_{\delta f}\right], \\
H(\delta f)=\frac{1}{2} \int d^{3} x d v\left[q \phi \delta f-\frac{M}{N_{0}} \frac{v}{F_{M}^{\prime}}\left(\delta f+\epsilon N_{0}^{\prime} x F_{M}\right)^{2}\right] .
\end{gathered}
$$

In Ref. [17], Hamiltonian closures have been derived for fluid systems originated by $\delta f$ models based on spatially homogeneous equilibrium distribution functions. Provided the above discussed boundary conditions are respected by the functional derivatives, the results of Ref. [17] can easily be extended to the system (61)-(34).

The Poisson bracket (62), expressed in terms of the moments, reads

$$
\{F, G\}=\sum_{m, n \in \mathbb{N}} \int d^{3} x\left[\left(\epsilon N_{0}^{\prime} P_{M m+n} x+\tilde{P}_{m+n}\right)\left[F_{m}, G_{n}\right]_{x}-\frac{N_{0}}{M}(m+n) P_{M m+n-1} F_{m} \partial_{z} G_{n}\right],
$$


where $P_{M i}=\int d v v^{i} F_{M}(v)$ for non-negative integers $i$.

Restricting to functionals of $\tilde{P}_{0}$ only, leads again to the Poisson bracket (29). Thus, also for the $\delta f$ model, the set of functionals of the zero order moment forms a sub-algebra.

Considering functionals of $\tilde{P}_{0}$ and $\tilde{P}_{1}$ leads to

$$
\begin{aligned}
& \{F, G\}=\int d^{3} x\left[\left(\epsilon N_{0}^{\prime} x+\tilde{P}_{0}\right)\left[F_{0}, G_{0}\right]_{x}+\tilde{P}_{1}\left(\left[F_{1}, G_{0}\right]_{x}+\left[F_{0}, G_{1}\right]_{x}\right)+\left(\epsilon N_{0}^{\prime} \frac{T_{i 0}}{M} x+\tilde{P}_{2}\right)\left[F_{1}, G_{1}\right]_{x}\right. \\
& \left.-\frac{N_{0}}{M}\left(F_{1} \partial_{z} G_{0}+F_{0} \partial_{z} G_{1}\right)\right],
\end{aligned}
$$

Following Ref. [17], and thus carrying out an analysis analogous to that recalled in Sec. III B, we obtain that the Hamiltonian closures for the fluid models derived from the bracket (65) correspond to

$$
\tilde{P}_{2}=\left(a-\frac{T_{i 0}}{M}\right) \epsilon N_{0}^{\prime} x+a \tilde{P}_{0},
$$

with $a$ arbitrary constant. Inserting the relation (66) into Eq. (65), yields the following Poisson bracket:

$$
\begin{aligned}
& \{F, G\}=\int d^{3} x\left[\left(\epsilon N_{0}^{\prime} x+\tilde{P}_{0}\right)\left[F_{0}, G_{0}\right]_{x}+\tilde{P}_{1}\left(\left[F_{1}, G_{0}\right]_{x}+\left[F_{0}, G_{1}\right]_{x}\right)+a\left(\epsilon N_{0}^{\prime} x+\tilde{P}_{0}\right)\left[F_{1}, G_{1}\right]_{x}\right. \\
& \left.-\frac{N_{0}}{M}\left(F_{1} \partial_{z} G_{0}+F_{0} \partial_{z} G_{1}\right)\right] .
\end{aligned}
$$

This bracket is also consistent with the criterion derived in Ref. [30], to construct 3D Poisson brackets for fluid plasma models assuming weak variations along the dominant component of the magnetic field. Indeed, such criterion states that brackets of the form

$$
\{F, G\}=\sum_{i, j, k=1}^{N} W_{k}^{i j} \int d^{3} x \chi^{k}\left[F_{i}, G_{j}\right]_{x}+\sum_{i, j=1}^{N} A^{i j} \int d^{3} x F_{i} \partial_{z} G_{j},
$$

between functionals $F\left(\chi^{1}, \cdots, \chi^{N}\right)$ and $G\left(\chi^{1}, \cdots, \chi^{N}\right)$, with integer $N$, are Poisson brackets if the constants $A^{i j}$ and $W_{k}^{i j}$ satisfy the relations

$$
A^{r s} W_{r}^{i j}=A^{r j} W_{r}^{s i}=A^{r i} W_{r}^{j s},
$$

where the sum over repeated indices is understood. In the case of the bracket (67) we have $N=2$ and the bracket can be written in the form (68) upon setting $\chi^{0}=P_{0}=\epsilon N_{0}^{\prime} x+\tilde{P}_{0}$ 
and $\chi^{1}=P_{1}=\tilde{P}_{1}$. The coefficients $W_{k}^{i j}$ and $A^{i j}$ then are all equal to zero apart from $W_{0}^{00}=1, W_{0}^{11}=a, W_{1}^{10}=W_{1}^{01}=1, A^{10}=A^{01}=-N_{0} / M$. It can easily be verified that such coefficients satisfy the relations (69).

When combined with the bracket (67), various choices for $a$ and for the Hamiltonian lead of course to various models. In particular, if one takes $a=0$ and

$$
H\left(\tilde{P}_{0}, \tilde{P}_{1}\right)=\frac{1}{2} \int d^{3} x\left(q \tilde{P}_{0} L_{H} \tilde{P}_{0}+\frac{M}{N_{0}} \tilde{P}_{1}^{2}\right)
$$

the resulting equations of motion can be written as

$$
\begin{aligned}
& \frac{\partial}{\partial t}\left(\frac{\Delta_{\perp} \phi}{B \Omega_{0}}-\frac{e}{T_{e 0}} \phi\right)-q\left[\phi, \frac{\Delta_{\perp} \phi}{B \Omega_{0}}\right]_{x}+\epsilon \frac{N_{0}^{\prime}}{N_{0}} \frac{c}{B} \frac{\partial \phi}{\partial y}-\frac{\partial \tilde{u}}{\partial z}=0, \\
& \frac{\partial \tilde{u}}{\partial t}-q[\phi, \tilde{u}]_{x}+\frac{q}{M} \frac{\partial \phi}{\partial z}=0
\end{aligned}
$$

where we identified $\tilde{P}_{0}=L_{H}^{-1} \phi$ and $\tilde{P}_{1}=N_{0} \tilde{u}$. The system (71)-(72) corresponds to the model presented in Ref. [31] and which describes drift modes coupled to ion-acoustic waves. Our procedure shows then that such model can be obtained, by means of a specific closure, from a Hamiltonian drift-kinetic equation, coupled to a quasi-neutrality relation and it also explicitly provides the Hamiltonian structure of the model.

\section{CONCLUSIONS}

We have shown that the drift-kinetic equation (2), coupled with the quasi-neutrality relation (3), which accounts for polarization effects, density and temperature gradients as well as adiabatic electrons, is a Hamiltonian system with a noncanonical Poisson structure. This result is based on symmetry and invertibility of the operator $L$ relating density fluctuations to the electrostatic potential, and relies on specific boundary conditions for the functional derivatives.

Subsequently, we considered the moments of the fluctuating part of the distribution function and addressed the question of whether, expressing the Poisson bracket (20) in terms of the moments $\left\{P_{i}\right\}_{i \in \mathbb{N}}$ and restricting the observables to functionals of the first

two moments, the resulting operation is still a Poisson bracket. For functionals of $\tilde{P}_{0}$ only, 
the answer is positive, because the set of such functionals turns out to form a sub-algebra. However, the resulting equation of motion, independently on the Hamiltonian, does not always correspond to the equation of motion obtained by directly taking the zero order moment of the drift-kinetic equation and imposing a functional relation $\tilde{P}_{1}=\mathcal{E}\left(\tilde{P}_{0}\right)$. This occurs only in the 2D limit and with a particular form for the Hamiltonian. In such limit, and assuming also weak spatial variations, one recovers the Poisson bracket and the Hamiltonian yielding the Charney-Hasegawa-Mima equation.

When extending the observables to functionals of $\tilde{P}_{0}$ and $\tilde{P}_{1}$, the resulting bilinear form is not a Poisson bracket in general, because it depends explicitly on $\tilde{P}_{2}$. We then looked for Hamiltonian closures, that is relations $\tilde{P}_{2}=\mathcal{F}\left(\tilde{P}_{0}, \tilde{P}_{1}\right)$ such that, when inserted into the bracket, the Jacobi identity is satisfied.

Extending the analysis of Ref. [17] to the case of equilibrium distribution function with spatial variations, we obtain that the only Hamiltonian closure is given by $P_{2}=P_{1}^{2} / P_{0}+\mathcal{A} P_{0}^{3}$, when expressed in terms of total moments. By means of this closure we obtain a Hamiltonian 3D fluid model evolving density and parallel velocity fluctuations and which, in general accounts for density and parallel equilibrium gradients and polarization drift. Because of the same form for the Poisson brackets, the models treated in Ref. [17] possess many features similar to those of the models presented here, such as for instance, the same Casimir functionals. This becomes evident provided, that in the present work one considers the total moments, given by the sum of the background and fluctuating components. In the present work, however, we consider a more complicated quasi-neutrality relation, for which we showed explicitly that the corresponding drift-kinetic model possesses a Hamiltonian structure. Such quasi-neutrality relation, which accounts for polarization terms, together with a drift-kinetic equation accounting for temperature and density gradients, can then yield models such as the Charney-Hasegawa-Mima equation, or generalizations of it, which were not derived in Ref. [17]. Also, here we treated in detail the case of the one-field models derived by functionals of the zero order moment only. The analysis showed that this case differs qualitatively from the case of the two-moment models, for the former leads 
to a sub-algebra, whereas the latter can be made Hamiltonian by applying a Hamiltonian closure.

We also considered the drift-kinetic model obtained from Eq. (2) after applying the $\delta f$ ordering and choosing, as equilibrium distribution function, a Maxwellian with weak spatial variations. In this case the set of functionals of the zero order moment forms again a subalgebra and leads to the Charney-Hasegawa-Mima equation. Considering as observables the functionals of the first two moments, on the other hand, requires a Hamiltonian closure for the bracket, which corresponds to imposing that the second order moment of the total distribution function $f$ be proportional, via an arbitrary constant $a$, to the zero order moment of $f$. In the case $a=0$, one recovers the Poisson bracket of the model derived by Meiss and Horton [31] to describe drift waves coupled to ion-acoustic waves.

Finally we remark that the Hamiltonian closure that we found might appear to be quite restrictive. This does not mean, however, that fluid models obtained with other closures are not Hamiltonian. A counterexample, in this respect, is provided by the model

$$
\frac{\partial \tilde{P}_{0}}{\partial t}=q\left[L \tilde{P}_{0}, \tilde{P}_{0}\right]_{x}-\partial_{z}\left(q L \tilde{P}_{0}+W\left(\tilde{P}_{0}\right)\right)
$$

which is obtained by taking the zero order moment of Eq. (61), setting $N_{0}=0$ and imposing a closure $\tilde{P}_{1}=q L \tilde{P}_{0}+W\left(\tilde{P}_{0}\right)$, for some function $W$. Eq. (73) possesses a Hamiltonian structure given by

$$
\begin{array}{r}
H\left(\tilde{P}_{0}\right)=\int d^{3} x\left(\frac{q}{2} \tilde{P}_{0} L \tilde{P}_{0}+\mathcal{W}\left(\tilde{P}_{0}\right)\right), \\
\{F, G\}=\int d^{3} x\left(\tilde{P}_{0}\left[F_{0}, G_{0}\right]_{x}-F_{0} \partial_{z} G_{0}\right),
\end{array}
$$

where $\mathcal{W}$ is such that $\mathcal{W}^{\prime}=W$.

The bracket (75), however, cannot be obtained from the bracket (64) by restricting to functionals of $\tilde{P}_{0}$, because, as above observed, this excludes the term in (75) depending on the $z$-derivative.

The bracket (75) should therefore be derived by means of a procedure different from the one we followed. The procedure used here, however, is systematic and guarantees, by 
construction, the Hamiltonian structure of the resulting fluid model. Adopting closures is in general an operation that imposes constraints on the original dynamics and that in general does not preserve the original Hamiltonian character of the dynamics. For closures other than the Hamiltonian closures, therefore, the Poisson bracket, if it exists, must be sought a posteriori, or more systematically, by applying other methods, such as for instance Dirac's theory of constraints, that guarantee that the Hamiltonian character of the system is preserved. Also, further Hamiltonian closures might be found by relaxing the assumption that the Hamiltonian closure be independent on the derivatives of the moments and contain no explicit dependence on the spatial coordinates.

[1] R.D. Hazeltine, Plasma Physics 15, 77 (1973).

[2] R.D. Hazeltine, A.A. Ware, Plasma Physics 20, 673 (1978).

[3] R.D. Hazeltine, J.D. Meiss, Plasma Confinement, Dover Publications, New York, (2003).

[4] K. Nishikawa, M. Wakatani, Plasma Physics, Springer, Heidelberg, (2000).

[5] S.I. Braginskii, Reviews of Plasma Physics 1, 205 (1965).

[6] G.W. Hammett, F.W. Perkins, Phys. Rev. Lett. 64, 3019 (1990).

[7] P.B. Snyder, G.W. Hammett, W. Dorland, Phys. Plasmas 4, 3974 (1997).

[8] T. Passot, P.L. Sulem, Phys. Plasmas 11, 5173 (2004).

[9] P. Goswami, T. Passot, P.L. Sulem, Phys. Plasmas 12, 2109 (2005).

[10] P. J. Morrison, Phys. Lett. 80A, 383 (1980).

[11] J. E. Marsden, A. Weinstein, Physica D 4, 394 (1982).

[12] B.A. Kupershmidt, J.I. Manin, Funktsional. Anal. i Prilozhen 12, 25 (1978).

[13] J. Gibbons, Physica D 3, 503 (1981).

[14] J. Gibbons, D.D. Holm, C. Tronci, Phys. Lett. A 372, 1024 (2008).

[15] J. Gibbons, D.D. Holm, C. Tronci, Phys. Lett. A 372, 4184 (2008).

[16] L. de Guillebon, C. Chandre, Phys. Lett. A 376, 3172 (2012).

[17] E. Tassi, J. Phys. A: Math. Theor. 47, 195501 (2014).

[18] V.Grandgirard, M. Brunetti, P. Bertrand, N. Besse, X. Garbet, P. Ghendrih, G. Manfredi, Y. Sarazin, O. Sauter, E. Sonnendrücker, J. Vaclavik, L. Villard, J. Comput. Phys. 217, 395 
(2006).

[19] P.J. Morrison, Phys. Plasmas 20, 012104 (2013).

[20] P.J. Morrison, M. Vittot, L. de Guillebon, Phys. Plasmas 20, 032109 (2013).

[21] P.J. Morrison, in Mathematical Methods in Hydrodynamics and Integrability in Dynamical Systems, AIP Conference Proceedings 88 La Jolla Institute, Ed. M. Tabor and Y.M. Treve, $13(1982)$.

[22] J.E. Marsden and T.R. Ratiu, Introduction to Mechanics and Symmetry, Springer-Verlag, Berlin (2002).

[23] P.J. Morrison, Rev. Mod. Phys. 70, 467 (1998).

[24] J.C. Charney, J. Atmos. Sci. 28, 1087 (1971).

[25] A. Hasegawa, K. Mima, Phys. Rev. Lett. 39, 205 (1977).

[26] A. Weinstein, Phys. Fluids 26, 388 (1983).

[27] E. Tassi, C. Chandre, P.J. Morrison, Phys. Plasmas 16, 082301 (2009).

[28] C. Chandre, E. Tassi, P.J. Morrison, Phys. Plasmas 17, 042307 (2010).

[29] B. Scott, Phys. Plasmas 17, 102306 (2010).

[30] E. Tassi, P.J. Morrison, D. Grasso, F. Pegoraro, Nucl. Fusion 50, 034007 (2010).

[31] J.D. Meiss, W. Horton, Phys. Fluids 26, 990 (1983).

[32] P. Morel, E. Gravier, N. Besse, R. Klein, A. Ghizzo, P. Bertrand, X. Garbet, P. Ghendrih, V. Grandgirard, Y. Sarazin, Phys. Plasmas 14, 112109 (2007). 